\title{
Cross-Sectional Characterization of Albumin Glycation State in Cerebrospinal Fluid and Plasma from Alzheimer's Disease Patients
}

\author{
M. Costa ${ }^{1}$, A. Mestre ${ }^{1}$, R. Horrillo ${ }^{1}$, A.M. Ortiz ${ }^{1}$, A. Pérez ${ }^{1}$, A. Ruiz $z^{2,3}$, M. Boada ${ }^{2,3}$, S. Grancha ${ }^{1}$

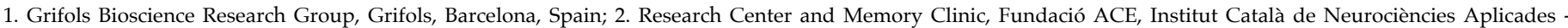

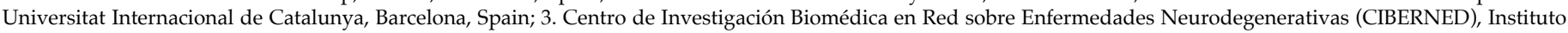
de Salud Carlos III, Madrid, Spain

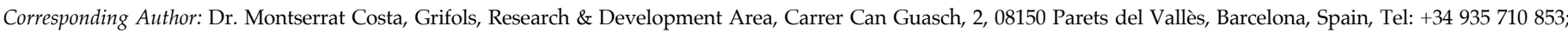
Fax: +34935 710 381, E-mail: montse.costa@grifols.com

\begin{abstract}
We determined albumin post-translational modifications (PTMs) by mass spectrometry (MS) in plasma and cerebrospinal fluid (CSF) from 31 Alzheimer's disease (AD) patients (with 27 samples of paired plasma-CSF from the same patients). Results were cross-sectionally compared with healthy controls. For percentage of relative intensity of glycated isoforms, plasma albumin was globally more glycated in AD patients than in healthy controls $(\mathrm{P}<0.01)$. MS results in plasma were confirmed by a quantitative enzymatic assay (Lucica GA-L) for albumin early-glycation detection. In CSF there were no global glycation differences detected by MS, although a different pattern of glycated isoforms was observed. Oxidized+glycated and cysteinylated+glycated isoforms were increased in both plasma and CSF of AD patients in comparison with healthy controls $(\mathrm{P}<0.001)$. Furthermore, AD patients showed higher glycation in plasma than in CSF $(\mathrm{P}<0.01)$. Our data support the role of glycation and oxidative stress in AD.
\end{abstract}

Key words: Albumin, glycation status, cerebrospinal fluid, plasma, Alzheimer's disease.

\section{Introduction}

A lbumin is the most abundant protein in the human body. In addition to its well-known role as a plasma expander, albumin has a number of relevant physiologic functions such as being the main extracellular antioxidant and transporter, and exerting metal-binding and scavenger activities (1). In albumin, glycation-induced conformational changes can have a deleterious effect in both its binding capacity (2-5) and antioxidant capacity (6-8). Elevated levels of glycated albumin are also associated with aging (9) and related conditions such as retinopathy, nephropathy, neuropathy, cardiovascular diseases and Alzheimer's disease $(\mathrm{AD})(4)$.

Serum albumin is thought to inhibit neuritic plaque formation tissue through regulating $\beta$-amyloid protein $(\mathrm{A} \beta)$ fibril growth in the brain (10), with glycated albumin being less effective than native albumin in preventing $A \beta$ aggregation (11). AD patients have been shown to have elevated levels of glycated proteins in serum $(12,13)$, plasma (14) and cerebrospinal fluid (CSF) $(15,16)$. More specifically, glycated albumin has been found increased in AD plasma and serum $(11,16)$, but no consistent results have been obtained in $\operatorname{AD~CSF~}(11,16)$. Although a paired comparison of protein glycation in plasma and CSF from the same AD patient has already been assessed (17), the same approach for albumin glycation has not been studied. In addition, to the best of our knowledge, a quantitative approach to specifically assess early-glycated albumin in AD plasma has not been addressed, despite the fact that the early glycation process has been reported as playing a role in $\mathrm{AD}$ (16).

In a previous paper we reported that albumin of $\mathrm{AD}$ patients was significantly more oxidized than in healthy subjects, with this effect more marked in CSF than in plasma (18). These data supported the involvement of oxidative stress in $\mathrm{AD}$, thus pointing out the need for further research to better understand the role of albumin in AD. The aim of this cross-sectional study was to extend the same approach to albumin glycation in plasma and CSF from AD patients, which was also characterized in comparison with age-matched healthy subjects.

\section{Methods}

\section{Experimental design}

Determination of albumin post-translational modifications (PTMs) for glycation in terms of their identification and relative quantification was performed by means of mass spectrometry (MS) technique. Albumin glycation levels were assessed in plasma $(n=28)$ and CSF $(n=30)$ samples from 31 patients with mild-moderate AD (there were 27 plasma and CSF samples drawn from the same individuals). Results were compared with albumin glycation levels assessed in plasma $(n=20)$ and CSF $(n=10)$ samples from healthy -not diagnosed with AD- agematched subject controls. Additionally, quantification of early-glycated albumin in plasma $(n=34$ for $A D$ 
patients; $n=31$ for controls) was measured by quantitative enzymatic assay (Lucica GA-L). Complementarily, the paired plasma versus CSF comparison of albumin glycation was performed in the 27 samples available from the same AD patients.

\section{Sampling}

Plasma and CSF samples taken from mild-moderate AD patients (53 to 78 years of age) meeting NINCDSADRDA criteria for probable AD, and Mini-Mental State Examination (MMSE) scores between $\geq 18$ and $\leq 26$ were baseline specimens provided by the centers participating in the clinical trial EudraCT \#2007-000414-36. Detailed patient population demographics, clinical profiles and inclusion/exclusion criteria are available in previous publications $(19,20)$.

Samples from control patients ( 48 to 81 years of age for plasma and 65 to 72 years of age for CSF) were kindly supplied by ACE Foundation, Catalan Institute of Applied Neurosciences (Barcelona, Spain) from their sample repository and purchased from Sera Laboratories International Ltd (West Sussex, UK), respectively.

All donors gave their informed consent in compliance with the Code of Ethics of the World Medical Association (Declaration of Helsinki) for studies involving humans.

Blood sampling (plasma) and lumbar puncture (CSF) were carried out following the standard techniques of each center (19). Albumin concentration in plasma and CSF from both AD patients and controls was similar (18).

\section{Liquid chromatography-Mass spectrometry}

PTMs of albumin were assessed on the intact mass protein (top-down) by using ultra high-performance liquid chromatography coupled to electrospray ionization mass spectrometer (LC-ESI-qTOF-MS) (21).

For plasma and CSF, albumin-enriched samples were obtained, separated by liquid chromatography and subsequently analyzed by MS on a Quadrupole Time of Flight analyzer with electrospray ionization source (ESI) (Agilent qTOF 6550 Jet Stream, Agilent Technologies, Santa Clara CA, USA). All procedures and mass spectrometry data analysis have been described in a previous publication (18). Albumin controls were processed in parallel to albumin-enriched samples to ensure sample stability during analysis.

Isoform relative intensity (\%) was calculated by dividing the isoform absolute intensity by the sum of all isoform absolute intensities, expressed as a percentage. For every comparison, a filter of frequency was applied to consider only those isoforms that were present in all analyzed samples of at least one of the compared groups.

The identification of albumin PTMs was performed as previously described (22-24) by analyzing 60,000-75,000 $\mathrm{Da}$ mass range and comparing the theoretical mass with the experimental value, allowing a mass drift of $10 \mathrm{Da}$ (Agilent BioConfirm software).

\section{Quantitative Enzymatic assay (Lucica GA-L)}

Early-albumin glycation (fructosyl groups bound to albumin) in plasma samples was determined by an in vitro diagnostic assay, Lucica ${ }^{\circledR}$ GA-L (25) (Asahi Kasei Pharma Corp., Tokio, Japan). This kit includes the measurement of glycated albumin by an enzymatic method using an albumin-specific protease and a ketoamine oxidase, and the measurement of total albumin using bromocresol purple reagent. Glycated albumin results were expressed in a percentage relative to the total albumin and corrected to match HPLC results: Glycated albumin $(\%)=($ glycated albumin $[\mathrm{g} / \mathrm{dL}] /$ total albumin $[\mathrm{g} / \mathrm{dL}]) / 1.14 \times 100+2.9$, following the instructions provided by the kit manufacturer. CSF analysis was not performed because the method's sensitivity is insufficient for low albumin concentration samples, such as those typical in CSF.

\section{Statistical analysis}

Variables are expressed as the median and interquartile range (IQR). Unpaired and paired Student's test and Mann-Whitney tests were used for the comparisons. Benjamini-Hochberg's correction for multiple comparisons was applied when comparing differences in albumin PTMs' relative abundance between groups (26).

To examine the magnitude of the differences in glycated albumin, the effect sizes based on standardized differences between mean scores were calculated (Cohen's delta) (27). Effect sizes of 0.20, 0.50 and 0.80 were considered small, medium and large, respectively (27).

Software used for charting and calculations was Graph-Pad Prism v6 (San Diego CA, USA) and GeneSpring 13.1 (Agilent Technologies, Santa Clara CA, USA).

\section{Results}

\section{Albumin glycation in plasma and CSF: AD patients versus controls}

Seventeen albumin PTMs were identified by intact mass protein analysis. In plasma, seven of these 17 PTMs corresponded to glycated PTMs: glycated $(\mathrm{Alb}+\mathrm{Glyc}) ;$ oxidized +glycated $\left(\mathrm{Alb}+\mathrm{SO}_{3} \mathrm{H}+\mathrm{Glyc}\right)$; cysteinylated+glycated+dehydroalanine $(\mathrm{Alb}+\mathrm{Cys}+\mathrm{Glyc}-$ DHA); cysteinylated+glycated (Alb+Cys+Glyc); two glycation modifications (Alb+2Glyc); cysteinylated+two glycation modifications (Alb+Cys+2Glyc); and three glycation modifications $\left(\mathrm{Alb}+{ }_{3} \mathrm{Glyc}\right)$. In CSF, four of these 17 albumin PTMs were identified as glycated PTMs: Alb+Glyc; $\mathrm{Alb}+\mathrm{SO}_{3} \mathrm{H}+\mathrm{Glyc} ; \mathrm{Alb}+\mathrm{Cys}+\mathrm{Glyc} ;$ and 
Alb+2Glyc.

MS values for percentage of relative intensity of albumin glycation in plasma showed that $\mathrm{AD}$ patients had higher amount of glycated albumin isoforms than controls when considering the sum of the seven glycated PTMs $(\mathrm{P}<0.01$, Cohen's delta: 1.0) (Figure 1a). Separately analyzed, four out of seven glycated PTMs showed a statistically significant increase in $\mathrm{AD}$ versus controls: oxidized $\left(\mathrm{Alb}+\mathrm{SO}_{3} \mathrm{H}+\mathrm{Glyc}[\mathrm{P}<0.001\right.$; Cohen's delta: 1.2]), and cysteinylated (Alb+Cys+Glyc-DHA [P $<0.0001$; Cohen's delta: 1.0], Alb+Cys+Glyc $[\mathrm{P}<0.0001 ;$ Cohen's delta: 1.7], and Alb+Cys+2Glyc $[\mathrm{P}<0.001$; Cohen's delta: 1.0]) (Figure 1b).

Figure 1. Albumin glycation in plasma as isoform relative intensity (\%) identified in Alzheimer's disease (AD) patients and healthy controls. A) Sum of all 7 glycated post-translational modifications (PTMs). B) The 7 glycated PTMs separately (glycated $[\mathrm{Alb}+\mathrm{Glyc}] ; \quad$ oxidized+glycated $\quad\left[\mathrm{Alb}+\mathrm{SO}_{3} \mathrm{H}+\mathrm{Glyc}\right]$; cysteinylated+glycated+dehydroalanine $[\mathrm{Alb}+\mathrm{Cys}+\mathrm{Glyc}-$ DHA]; cysteinylated+glycated [Alb+Cys+Glyc]; two glycation modifications $[\mathrm{Alb}+2 \mathrm{Glyc}]$; cysteinylated+two glycation modifications [Alb+Cys+2Glyc]; three glycation modifications $[\mathrm{Alb}+3 \mathrm{Glyc}])$

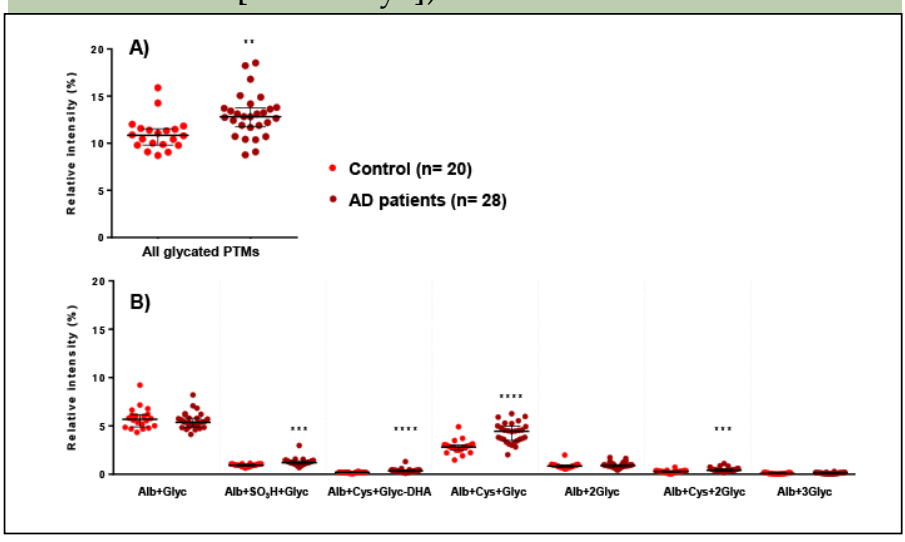

Data are shown as median and IQR $\left({ }^{* *}, \mathrm{p}<0.01 ;{ }^{* * *}, \mathrm{p}<0.001 ;{ }^{* * * *}, \mathrm{p}<0.0001 ; \mathrm{AD}\right.$ versus control).

MS results in plasma were confirmed by the quantitative enzymatic assay (Lucica GA-L) for albumin early-glycation detection. The percentage of glycated plasma albumin in $\mathrm{AD}$ patients was $12.9 \%$ (12.3\%-14.9\%) versus $11.6 \%(10.5 \%-13.4 \%)$ in controls (median-IQR; $\mathrm{P}<0.001$; Cohen's delta: 0.7).

MS values of albumin glycation in CSF showed no differences in percentage of relative intensity when comparing the sum of the four glycated PTMs in AD patients versus controls (Figure 2a). However, if separately analyzed, three out of four glycated PTMs showed statistically significant differences in $A D$ versus controls: a decrease in Alb+Glyc isoform $(\mathrm{P}<0.0001$; Cohen's delta: 1.7) and an increase in the oxidized and cysteinylated forms $\left(\mathrm{Alb}+\mathrm{SO}_{3} \mathrm{H}+\mathrm{Glyc}[\mathrm{P}<0.0001\right.$ : Cohen's delta: 3.0$]$ and $\mathrm{Alb}+\mathrm{Cys}+\mathrm{Glyc}[\mathrm{P}<0.0001$ : Cohen's delta: 1.8]) (Figure 2b).
Figure 2. Albumin glycation in cerebrospinal fluid (CSF) as isoform relative intensity (\%) identified in Alzheimer's disease $(\mathrm{AD})$ patients and healthy controls. A) Sum of 4 glycated PTMs. B) The 4 glycated PTMs separately (glycated $[\mathrm{Alb}+\mathrm{Glyc}]$; oxidized+glycated $\left[\mathrm{Alb}+\mathrm{SO}_{3} \mathrm{H}+\mathrm{Glyc}\right]$; cysteinylated+glycated [Alb+Cys+Glyc]; two glycation modifications [Alb+2Glyc])
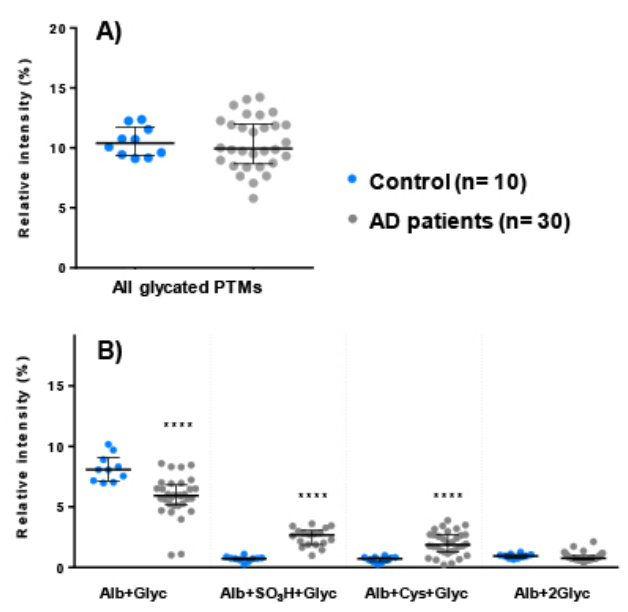

Data are shown as median and IQR (****, $\mathrm{p}<0.0001 ; \mathrm{AD}$ versus control).

\section{Albumin glycation in AD patients: plasma versus CSF}

When comparing albumin glycated PTMs in the subset of $27 \mathrm{AD}$ samples consisting of paired plasma and CSF specimens both obtained from the same patient, plasma albumin was found significantly more glycated than in their paired CSF samples (Table 1). Moreover, the specific analysis showed that six out of seven glycated PTMs of albumin were statistically significantly different between compartments. The most relevant ones were $\mathrm{Alb}+\mathrm{Glyc}$ and $\mathrm{Alb}+\mathrm{Cys}+\mathrm{Glyc}$ in plasma, and Alb+Glyc and $\mathrm{Alb}+\mathrm{SO}_{3} \mathrm{H}+\mathrm{Glyc}$ in CSF. Details are shown in Table 1.

\section{Discussion}

In this study we observed that plasma albumin was overall more glycated in $\mathrm{AD}$ patients than in healthy agematched controls, when analyzed as the percentage of relative intensity of albumin glycation isoforms assessed by MS. Conversely, in CSF albumin, there were no global glycation differences, although a different pattern of glycation isoforms was observed. Moreover, AD patients had their albumin significantly more glycated in plasma than in CSF.

In plasma samples from $\mathrm{AD}$ patients, when considering all the glycated albumin PTMs separately, statistical significance versus controls was shown with the oxidized+glycated albumin forms and especially the cysteinylated+glycated albumin forms, rather than 
Table 1. Albumin glycation in paired samples of plasma and cerebrospinal fluid (CSF) of Alzheimer's disease (AD) patients $(\mathrm{n}=27)$. Compared relative intensity $(\%)$ of 7 individual glycated post-translational modifications (PTMs) (glycated $[\mathrm{Alb}+\mathrm{Glyc}]$; oxidized+glycated $\left[\mathrm{Alb}+\mathrm{SO}_{3} \mathrm{H}+\mathrm{Glyc}\right]$; cysteinylated+glycated+dehydroalanine $[\mathrm{Alb}+\mathrm{Cys}+\mathrm{G}-$ lyc-DHA]; cysteinylated+glycated [Alb+Cys+Glyc]; two glycation modifications [Alb+2Glyc]; cysteinylated+two glycation modifications [Alb+Cys+2Glyc]; three glycation modifications [Alb+3Glyc]); and the sum of all PTMs are shown (median and IQR)

\begin{tabular}{|l|c|c|c|c|}
\hline Glycated PTM & Plasma AD (\%) & CSF AD (\%) & p value & Cohen's delta \\
\hline Alb+Glyc & $5.4[4.9-5.8]$ & $6.0[5.3-7.0]$ & $<0.05$ & 0.6 \\
\hline Alb+SO 3 H+Glyc & $1.2[1.1-1.4]$ & $2.7[1.9-3.0]$ & $<0.0001$ & 2.2 \\
\hline Alb+Cys+Glyc-DHA & $0.37[0.27-0.42]$ & $0.88[0.67-1.1]$ & $<0.0001$ & 1.6 \\
Alb+Cys+Glyc & $4.5[3.6-5.0]$ & $1.8[1.3-2.7]$ & $<0.0001$ & 2.3 \\
Alb+2Glyc & $0.90[0.82-1.0]$ & $0.77[0.64-1.1]$ & $>0.05$ & 0.2 \\
Alb+Cys+2Glyc & $0.42[0.37-0.56]$ & $0.27[0.18-0.38]$ & $<0.0001$ & 1.1 \\
Alb+3Glyc & $0.11[0.06-0.14]$ & $0.20[0.15-0.28]$ & $<0.05$ & 1.2 \\
\hline All glycated PTMs & $13[12-14]$ & $11[9.8-14]$ & $<0.01$ & 0.4 \\
\hline
\end{tabular}

with the purely glycated albumin. Since cysteinylated forms are considered to be reversibly oxidized albumin modifications (1), these findings would support the higher content of reversibly oxidized albumin (HNA1) detected by HPLC-FLD analysis in AD plasma samples compared to control plasma (18). Interestingly, cysteinylated albumin has been described as a sensitive plasma marker in oxidative stress-related chronic diseases (28).

In CSF, overall albumin glycation levels were similar between $\mathrm{AD}$ patients and controls but, interestingly, the glycation pattern was different. AD patients showed lower content of glycated albumin but increased content of both the oxidized+glycated and cysteinylated+glycated albumin forms. Again, since cysteinylated forms are considered to be reversibly oxidized albumin modifications while $\mathrm{SO}_{3} \mathrm{H}$ forms are considered to be irreversibly oxidized albumin modifications (2), these findings are in accordance to a higher content in HNA1 and irreversibly oxidized albumin (HNA2) observed by HPLC-FLD analysis in AD CSF (18).

Furthermore, MS results obtained in plasma from $\mathrm{AD}$ patients and healthy controls were confirmed by a quantitative enzymatic assay for early glycation detection of albumin (Lucica GA-L).

Albumin glycation in AD patients has been previously addressed by a few other authors. Increased levels of early glycation products in all major proteins of CSF of $\mathrm{AD}$ patients, including albumin, apolipoprotein $\mathrm{E}$ and transthyretin, have been reported (16). Furthermore, targeted assessment of advanced glycation end products in albumin has been addressed in plasma (11) and serum (13), showing higher levels than in a control population, while presenting no differences in CSF (11). Our findings in CSF albumin glycation are in accordance with those obtained by Ramos-Fernández et al. (11). Differences in the AD study population, as well as in the experimental approach, might explain the lack of consensus with all of the investigations.

Based on our literature search, albumin glycation in paired plasma and CSF samples taken from the same patient has not been previously reported. Our results showed higher overall albumin glycation in plasma compared to CSF, with both compartments being different in terms of PTMs relative quantification. Besides the glycated albumin form, in plasma the reversibly oxidized cysteinylated+glycated albumin was relevant, while in CSF the irreversibly oxidized $\mathrm{SO}_{3} \mathrm{H}+$ glycated isoform gained predominance. These results suggest a restriction of albumin communication in both compartments, which would present different environments in terms of glycation and oxidation.

To summarize, accepting the limitations of this study, such as the sample size and the use of MS as a semiquantitative method to analyze albumin glycation, the results presented here suggest that in both plasma and CSF samples taken from mild to moderate AD patients there is a marked increase in the cysteinylated+glycated and oxidated+glycated albumin forms, compared to healthy age-matched controls. Furthermore, AD patients showed higher glycation in plasma than in CSF. These data support the role of glycation and oxidative stress in $\mathrm{AD}$ and deserve further investigation.

\footnotetext{
Acknowledgements: We thank Pol Herrero (Proteomics facility of the Centre for Omic Sciences [COS] Joint Unit of the Universitat Rovira i Virgili-Eurecat) for his contribution to the mass spectrometry analysis. Cristina Aparicio, Francisca Doncel, Santiago Garcia, Carlota Gelabert, Aida Raventós, Ana María Siles, Jordi Vidal, and Eva Vior (Grifols) are acknowledged for their expert technical assistance. Jordi Bozzo PhD, CMPP (Grifols) is acknowledged for medical writing and editorial support in the preparation of this manuscript.
}

Conflict of interest: MC, AM, RH, AMO, AP and SG are employees of Grifols, a manufacturer of plasma derivatives. MB has consulted for Araclon, Avid, Grifols, Lilly, Nutricia, Roche and Servier. She received fees for lectures, and/or reimbursement of expenses for congresses attendance, and/or funds for research from Araclon, Grifols, Nutricia, Roche and Servier. She has not received personal compensations from these organizations. AR has consulted for Grifols. He is a member of the scientific advisory board of Landsteiner Genmed. He received funds for research from Araclon, Grifols, Nutricia, Roche and Servier. He received reimbursement of expenses for congresses attendance from Araclon, Grifols, and 
Landsteiner Genmed. He has not received personal compensations from these organizations.

Ethical standards: All donors gave their informed consent in compliance with the Code of Ethics of the World Medical Association (Declaration of Helsinki) for studies involving humans.

Open Access: This article is distributed under the terms of the Creative Commons Attribution 4.0 International License (http:/ / creativecommons.org/ licenses/by/4.0/), which permits use, duplication, adaptation, distribution and reproduction in any medium or format, as long as you give appropriate credit to the original author(s) and the source, provide a link to the Creative Commons license and indicate if changes were made.

\section{References}

1. Colombo G, Clerici M, Giustarini D, Rossi R, Milzani A, Dalle-Donne I. Redox albuminomics: oxidized albumin in human diseases. Antioxid Redox Signal 2012;17, 1515-1527.

2. Anguizola J, Matsuda R, Barnaby OS, Hoy KS, Wa C, DeBolt E, Koke M, Hage DS. Review: Glycation of human serum albumin. Clinica Chimica Acta 2013;425, 64-76.

3. Dozio E, Di Gaetano N, Findeisen P, Corsi Romanelli MM. Glycated albumin: from biochemistry and laboratory medicine to clinical practice. Endocrine 2016;55, 682-690.

4. Rondeau P, Bourdon E. The glycation of albumin: Structural and functional impacts. Biochimie 2011;93, 645-658.

5. Vetter SW. in Advances in Clinical Chemistry Elsevier, 2015; pp. 205-275

6. Baraka-Vidot J, Guerin-Dubourg A, Dubois F, Payet B, Bourdon E, Rondeau P. New insights into deleterious impacts of in vivo glycation on albumin antioxidant activities. Biochim Biophys Acta 2013;1830, 3532-3541.

7. Khan MS, Tabrez S, Rabbani N, Shah A. Oxidative Stress Mediated Cytotoxicity of Glycated Albumin: Comparative Analysis of Glycation by Glucose Metabolites. J Fluorescence 2015;25, 1721-1726.

8. Rondeau P, Singh NR, Caillens H, Tallet F, Bourdon E. Oxidative stresses induced by glycoxidized human or bovine serum albumin on human monocytes. Free Radic Biol Med 2008;45, 799-812.

9. Arasteh A, Farahi S, Habibi-Rezaei M, Moosavi-Movahedi A. Glycated albumin: an overview of the In Vitro models of an In Vivo potential disease marker. J Diabetes Metab Disord 2014;13, 49.

10. Stanyon HF, Viles JH. Human Serum Albumin Can Regulate Amyloid- $\beta$ Peptide Fiber Growth in the Brain Interstitium. J Biol Chem 2012;287, 2816328168.

11. Ramos-Fernández E, Tajes M, Palomer E, Ill-Raga G, Bosch-Morató M, Guivernau B, Román-Dégano I, Eraso-Pichot A, Alcolea D, Fortea J, Nuñez L, Paez A, Alameda F, Fernández-Busquets X, Lleó A, Elosúa R, Boada M, Valverde MA, Muñoz FJ. Posttranslational Nitro-Glycative Modifications of Albumin in Alzheimer's Disease: Implications in Cytotoxicity and Amyloid- $\beta$ Peptide Aggregation. J Alzheimers Dis 2014;40, 643-657.

12. Lin CY, Sheu JJ, Tsai IS, Wang ST, Yang LY, Hsu IU, Chang HW, Lee HM, Kao $\mathrm{SH}$, Lee CK, Chen CH, Lin YF. Elevated IgM against Nepsilon-(Carboxyethyl) lysine-modified Apolipoprotein A1 peptide 141-147 in Taiwanese with Alzheimer's disease. Clin Biochem 2018;56, 75-82.

13. Meli M, Perier C, Ferron C, Parssegny F, Denis C, Gonthier R, Laurent B, Reynaud E, Frey J, Chamson A. Serum pentosidine as an indicator of Alzheimer's disease. J Alzheimers Dis 2002;4, 93-96.
14. Riviere S, Birlouez-Aragon I, Vellas B. Plasma protein glycation in Alzheimer's disease. Glycoconj J 1998;15, 1039-1042.

15. Ahmed N, Ahmed U, Thornalley PJ, Hager K, Fleischer G, Munch G. Protein glycation, oxidation and nitration adduct residues and free adducts of cerebrospinal fluid in Alzheimer's disease and link to cognitive impairment. J Neurochem 2005;92, 255-263.

16. Shuvaev VV, Laffont I, Serot J-M, Fujii J, Taniguchi N, Siest G. Increased protein glycation in cerebrospinal fluid of Alzheimer's disease. Neurobiol Aging 2001;22, 397-402.

17. Bar KJ, Franke S, Wenda B, Muller S, Kientsch-Engel R, Stein G, Sauer H. Pentosidine and N(epsilon)-(carboxymethyl)-lysine in Alzheimer's disease and vascular dementia. Neurobiol Aging 2003;24, 333-338.

18. Costa M, Horrillo R, Ortiz AM, Perez A, Mestre A, Ruiz A, Boada M, Grancha S. Increased Albumin Oxidation in Cerebrospinal Fluid and Plasma from Alzheimer's Disease Patients. J Alzheimers Dis 2018;63, 1395-1404.

19. Boada M, Anaya F, Ortiz P, Olazaran J, Shua-Haim JR, Obisesan TO, Hernandez I, Munoz J, Buendia M, Alegret M, Lafuente A, Tarraga L, Nunez L, Torres M, Grifols JR, Ferrer I, Lopez OL, Paez A. Efficacy and Safety of Plasma Exchange with 5\% Albumin to Modify Cerebrospinal Fluid and Plasma Amyloid-beta Concentrations and Cognition Outcomes in Alzheimer's Disease Patients: A Multicenter, Randomized, Controlled Clinical Trial. J Alzheimers Dis 2017;56, 129-143.

20. Cuberas-Borrós G, Roca I, Boada M, Tárraga L, Hernández I, Buendia M, Rubio L, Torres G, Bittini A, Guzmán de Villoria J, Pujadas F, Torres M, Núñez L, Castell J, Páez A. Longitudinal neuroimaging analysis in mildmoderate Alzheimer's disease patients treated with plasma exchange with 5\% human albumin. J Alzheimers Dis 2018;61, 321-332.

21. Kawakami A, Kubota K, Yamada N, Tagami U, Takehana K, Sonaka I, Suzuki E, Hirayama K. Identification and characterization of oxidized human serum albumin. A slight structural change impairs its ligand-binding and antioxidant functions. FEBS J 2006;273, 3346-3357.

22. Naldi M, Giannone FA, Baldassarre M, Domenicali M, Caraceni P, Bernardi $\mathrm{M}$, Bertucci C. A fast and validated mass spectrometry method for the evaluation of human serum albumin structural modifications in the clinical field. Eur J Mass Spectrom 2013;19, 491-496.

23. Domenicali M, Baldassarre M, Giannone FA, Naldi M, Mastroroberto M, Biselli M, Laggetta M, Patrono D, Bertucci C, Bernardi M, Caraceni P. Posttranscriptional changes of serum albumin: clinical and prognostic significance in hospitalized patients with cirrhosis. Hepatology 2014;60, 18511860.

24. Bar-Or R, Rael LT, Bar-Or D. Dehydroalanine derived from cysteine is a common post-translational modification in human serum albumin. Rapid Commun Mass Spectrom 2008;22, 711-716.

25. Kohzuma T, Yamamoto T, Uematsu Y, Shihabi ZK, Freedman BI. Basic performance of an enzymatic method for glycated albumin and reference range determination. J Diabetes Sci Technol 2011;5, 1455-1462.

26. Benjamini $Y$, Heller R. Screening for partial conjunction hypotheses. Biometrics 2008;64, 1215-1222.

27. Cohen J. Statistical Power Analysis for the Behavioral Sciences, Hillsdale, N.J. : Lawrence Erlbaum Associates, 1988.

28. Nagumo K, Tanaka M, Chuang VT, Setoyama H, Watanabe H, Yamada N, Kubota K, Tanaka M, Matsushita K, Yoshida A, Jinnouchi H, Anraku M, Kadowaki D, Ishima Y, Sasaki Y, Otagiri M, Maruyama T. Cys34-cysteinylated human serum albumin is a sensitive plasma marker in oxidative stress-related chronic diseases. PLoS One 2014;9, e85216. 